\title{
Analyse comparée du contenu du premier traité de physique canadien
}

\section{Alain Couillard}

\section{RÉSUMÉ}

Joseph-Édouard Cauchon rédige en 1841 les Notions élémentaires de physique, premier manuel de physique canadien. Inspiré de manuels français et de l'enseignement scientifique donné au Séminaire de Québec, ce traité de 124 pages contient des connaissances pratiques destinées, selon son auteur, à mousser les jeunes talents pour les arts usuels. Afin d'évaluer la portée des Notions, l'article présente une analyse comparée de ce traité en lien avec le contenu et la forme de manuels français et américains. Si les auteurs de traités destinés aux collèges royaux français sont fervents de précision et de savoirs abstraits, leurs homologues américains rédigeant des manuels pour les high schools semblent plus pragmatiques et plus favorables aux connaissances appliquées. Les racines de ces différences se situent dans les conditions institutionnelles et pédagogiques de l'utilisation des manuels. C'est dans ces mêmes conditions que semble résider l'échec des Notions : trop simples pour les collèges et trop complexes pour les écoles élémentaires.

\section{ABSTRACT}

In 1841, Joseph-Édouard Cauchon wrote Notions élémentaires de physique, the first Canadian physics textbook. Inspired by French texts and by the teaching of science at the Séminaire de Québec, his 124-page treatise contains practical knowledge intended, according to its author, to inspire future scientists. In order to evaluate the impact of Notions, this article presents a comparative analysis of the book in relation to the form and content of French and American textbooks. While the authors of books destined for use in French collèges royaux are fervent about precision and abstract knowledge, their American counterparts, writing for high school students, seem to be both more pragmatic and more interested in applied knowledge. The roots of these differences are found in the educational and institutional contexts in which the textbooks were used. The failure of Notions can also be explained by context: its content was too simple for use in secondary schools and too complex for elementary students.

En 1841, à l'orée d'une longue carrière journalistique et politique, le jeune JosephÉdouard Cauchon (1816-1885) rédige le premier manuel de physique canadien intitulé Notions élémentaires de physique. ${ }^{1}$ Destiné aux écoles et au public en général, son traité expose en 124 pages les principaux concepts de cette science. Le présent 
article traitera spécifiquement du contenu des Notions en comparaison avec les manuels français et américains parus à la même époque. En effet, le premier manuel de physique rédigé par un Canadien anglais, John Herbert Sangster, paraît vingt ans après celui de Cauchon sous le titre de Natural Philosophy (1861). ${ }^{2}$ Associé à l'école normale de Toronto qui forme les professeurs pour l'enseignement élémentaire, Sangster est aussi l'auteur de traités d'arithmétique et d'algèbre. ${ }^{3}$ En raison de l'écart dans le temps séparant les Notions du traité de Sangster, nous avons toutefois jugé qu'il n'était pas pertinent de l'intégrer à notre étude, d'où l'absence du Haut-Canada dans notre comparaison.

Au-delà des repères permettant d'évaluer l'état des connaissances du traité de Cauchon, notre recherche mettra en relief les différentes manières de concevoir l'enseignement de la physique en France, aux États-Unis et au Bas-Canada. Plus qu'une simple étude comparative, notre enquête traitera des rapports entre le style et le contenu des manuels et les conditions institutionnelles et pédagogiques de leur utilisation.

Les travaux portant spécifiquement sur l'histoire des manuels scolaires québécois sont peu nombreux. À l'automne 2006, la publication du collectif 300 ans de manuels scolaires au Québec, dirigé par Paul Aubin, attirait l'attention sur le vide qui reste à combler en ce domaine. Accompagnant une exposition du même nom présentée à la Bibliothèque nationale du Québec, cet ouvrage montre en quoi les manuels, qui constituent de véritables " miroirs de la société ", ${ }^{4}$ devraient intéresser davantage les historiens. Le vide est particulièrement perceptible dans l'historiographie sur les manuels de sciences. Datant déjà de quelques décennies, les travaux de Claude Galarneau sur le cours classique ont posé des balises permettant de repérer les traités employés par les professeurs au fil du temps. ${ }^{5}$ Dans un cadre plus large, les auteurs d'Histoire des sciences au Québec présentent divers manuels-dont les Notions élémentaires de physique - en les insérant dans une généalogie de l'enseignement scientifique. ${ }^{6}$ Malgré l'existence de ces quelques points de repère, un travail considérable demeure toutefois à accomplir sur le contenu, les caractéristiques et le contexte de rédaction des premiers traités de sciences bas-canadiens de la première moitié du $19^{\mathrm{e}}$ siècle.

En France, l'étude la plus pertinente parue à ce jour sur les manuels de physique est l'oeuvre de Claudette Balpe, Enseigner la physique au collège et au lycée.7 L'auteure retrace l'évolution des pratiques professorales en accordant autant d'importance aux méthodes d'enseignement qu'au contenu enseigné. Elle offre également une généalogie des plans et des contenus des manuels qui ont été les plus utilisés dans les lycées à partir du $18^{\mathrm{e}}$ siècle. Du côté américain_bien que moins récent_l'ouvrage de John Alfred Nietz, The Evolution of American Secondary School Textbooks, ${ }^{8}$ analyse le contenu des manuels de physique employés dans les académies et les high schools américains au cours du $19^{\mathrm{e}}$ siècle. À partir du recensement exhaustif de 45 manuels distincts, Nietz calcule l'espace moyen consacré à chacun des grands thèmes de la physique. Il s'intéresse aussi à la présence de divers éléments didactiques.

En plus de servir de cadres pour nos analyses, ces études offriront des points de comparaison utiles pour l'évaluation des Notions élémentaires de physique. 


\section{Joseph Cauchon et le contexte de rédaction des Notions élémentaires}

Cauchon suit son cours classique au Séminaire de Québec entre 1830 et 1839.9 Durant les deux dernières années de son cursus, constituées des classes de philosophie I et II, il reçoit un enseignement scientifique condensé comprenant des éléments de physique, de chimie et de diverses sciences naturelles. ${ }^{10}$ Le professeur de physique est alors Louis-Jacques Casault. Selon le descriptif de son cours, il fonde son enseignement sur les notes d'un ancien professeur (Jérôme Demers) et sur un manuel de physique d'un auteur français de renom, Eugène Péclet. ${ }^{11}$ Bénéficiant des améliorations faites au cours par son prédécesseur, Casault s'appuie sur 1117 pages de notes très actuelles où des thèmes comme la mécanique, l'optique, l'électricité et la chaleur sont développés avec moult détails. ${ }^{12}$ En classe, selon la mode de l'époque, le professeur dicte ses notes aux élèves qui les recopient dans leur cahier. À l'occasion, pour illustrer certains phénomènes, il emprunte des instruments aux cabinets de physique qui sont progressivement constitués dans les collèges. Précisons toutefois qu'en l'absence de programmes communs à l'échelle de la province, la qualité de l'enseignement des sciences dans les quelques collèges du Bas-Canada est souvent tributaire des compétences et de la motivation du professeur ayant la responsabilité de cet enseignement. ${ }^{13}$

Deux ans après sa sortie du Séminaire, au moment où il rédige les Notions élémentaires, Cauchon occupe provisoirement le poste de rédacteur pour le journal Le Canadien. ${ }^{14}$ Âgé de 25 ans, il mène, en parallèle à son travail, des études en droit auprès d'un notaire reconnu de la ville de Québec. ${ }^{15}$ Bien que les sources sur la première portion de sa vie soient peu nombreuses, il semble que Cauchon s'implique activement dans la vie intellectuelle et culturelle de Québec. ${ }^{16}$ On sait entre autres qu'il participe à des réunions en vue de créer un institut littéraire et scientifique dans le vieux collège des Jésuites (projet qui ne verra pas le jour). Il y côtoie des membres influents de l'élite canadienne-française tels Napoléon Aubin et Étienne Parent. ${ }^{17}$ En raison de son travail au Canadien, il est également au courant des sujets d'actualité. Dans ce périodique publié trois fois par semaine, les nouvelles provinciales et internationales sont tirées de journaux canadiens et étrangers, et une large place est accordée aux activités municipales, économiques et littéraires de la région de Québec. On y retrouve aussi certains articles à caractère scientifique provenant de la presse étrangère. Il s'agit la plupart du temps d'annonces d'inventions et de découvertes, ainsi que de résumés de réunions tenues par des sociétés savantes. ${ }^{18}$

Si nos informations sur le jeune Cauchon (avant 1841) s'arrêtent là, rappelons toutefois que la première moitié du $19^{\mathrm{e}}$ siècle est, pour le Bas-Canada, une période riche en bouleversements autant politiques que socioculturels. De 1815 à 1838, le nombre de personnes exerçant une profession libérale triple. ${ }^{19}$ Une aisance nouvelle contribue à l'émergence d'un " goût " pour les sciences et les arts. ${ }^{20}$ Le commerce de biens et de personnes entre le Vieux Continent et le Nouveau reprend après le blocus napoléonien, profitant aux libraires qui importent des livres de natures diverses en grande quantité. ${ }^{21}$ À Montréal et à Québec, on voit la formation d'associations 
savantes se voulant lieux d'échanges et de divertissement. De nature patriotique avant les événements de 1837-1838, ces associations se rapprochent par la suite des associations savantes anglophones pour traiter davantage de sciences et de culture. ${ }^{22}$ Des projets de bibliothèques, de musées, de grands instituts culturels sont proposés (même si certains ne se concrétisent pas). L'essor de la presse permet la circulation des idées, des opinions provenant non seulement du pays, mais aussi de l'étranger.

Dans les sphères de la petite bourgeoisie professionnelle francophone que côtoie Cauchon, une des idéologies en émergence est le libéralisme économique. Accompagnant les débuts de l'ère industrielle, cette idéologie est axée sur l'individu et ses libertés. Il importe de donner à chacun les outils de sa réussite afin de favoriser la prospérité du Bas-Canada, affligé par des conflits qui créent un relatif climat de stagnation. Comme le note Yvan Lamonde : "Pour les jeunes qui ont vingt ans en 1840 , l'ignorance est intenable devant le développement commercial et culturel ${ }^{23}$

L'éducation est donc une des voies envisagées pour mieux outiller la jeunesse pour l'avenir. La loi scolaire de 1829, qui prévoit un accroissement du financement gouvernemental pour les écoles, est un premier pas vers la démocratisation de l'éducation élémentaire. En 1828, on recensait 325 écoles et 11679 écoliers au Bas-Canada. Trois ans plus tard, ces nombres passent à 1216 et $41821 .{ }^{24}$ En outre, le nombre de collèges passe de 4 à 8 entre 1825 et 1844 avec la fondation des collèges de Sainte-Thérèse (1825), de La Pocatière (1829), de L'Assomption (1832) et de Chambly (1825). ${ }^{25}$

Au-delà des questions de la simple accessibilité à l'éducation, certains membres de la petite bourgeoisie professionnelle se préoccupent de plus en plus du contenu des programmes scolaires. À partir des années 1840, un discours en faveur d'un enseignement plus appliqué émerge :

La prospérité d'un pays, sera nécessairement proportionnée à l'industrie individuelle et collective de ceux qui l'habitent. Le succès du cultivateur, du marchand et du commerçant, de l'ouvrier, du marin, en un mot le succès de tous, dépend de leur connaissance de l'art, de la profession ou du métier qu'ils suivent, et par conséquent la prospérité et le bonheur de la société et des individus, dépendent essentiellement du degré d'intelligence et de connaissances pratiques qui existent dans un pays. ${ }^{26}$

Cette demande nouvelle pour un enseignement pratique, combinée à l'augmentation de la clientèle scolaire, bénéficie grandement au secteur de l'édition de manuels. L'essor de la presse ayant créé un contexte favorable pour des imprimeurs-éditeurs aux assises solides (comme Duvernay-Fabre à Montréal), ${ }^{27}$ les portes des ateliers d'impression s'ouvrent aux auteurs scolaires bas-canadiens avec des perspectives alléchantes de profits. L'intérêt est réciproque puisque l'adoption de nouvelles lois sur la propriété littéraire assure aux auteurs des revenus plus substantiels qu'auparavant. ${ }^{28} \mathrm{Ces}$ conjonctures se traduisent par un accroissement significatif du nombre de publications de manuels de sciences au Bas-Canada à partir des années 1830. C'est dans ce courant que s'inscrit la publication des Notions élémentaires de physique (fig. 1).

Fait à préciser, les premiers traités de sciences bas-canadiens apparaissent pour la 


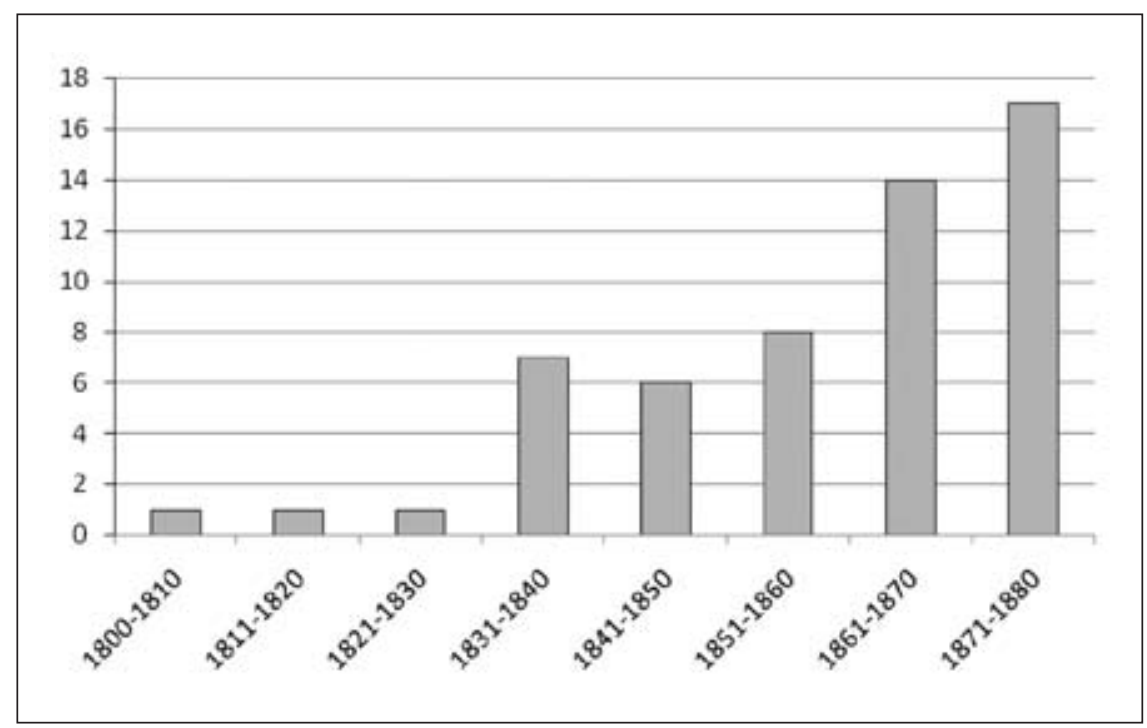

Figure 1 : Parution des manuels de sciences canadiens rédigés en français par périodes

plupart en marge du système d'éducation. Tout comme Cauchon, les auteurs sont souvent de jeunes autodidactes exerçant une profession libérale. ${ }^{29}$ On peut présumer qu'ils sont particulièrement sensibles à l'enthousiasme nouveau pour le progrès, l'éducation et les connaissances pratiques. En plus des avantages financiers tirés de la publication de manuels, ces jeunes auteurs souhaitent probablement promouvoir, à hauteur de leurs moyens, le savoir scientifique qu'ils ont acquis de leur formation classique ou de leurs lectures personnelles.

\section{Les sources d'inspiration des Notions élémentaires de physique}

Au moment où il annonce dans Le Canadien la mise en vente officielle des Notions, Cauchon fournit les informations suivantes sur son public cible et ses sources d'inspiration :

Nous avons encore promis d'être clairs afin de nous mettre autant que possible à la portée de tous les lecteurs : ce à quoi nous avons tâché d'atteindre au risque de nous rendre trivial. Mais comme nous avions également pour souscripteurs et les écoles ordinaires et des institutions supérieures, nous n'avons pu quelquefois nous contenter du simple exposé des phénomènes, [...] nous n'avons pas prétendu faire un ouvrage de notre composition proprement dite, nous avons recueilli ces principes élémentaires dans les auteurs les plus recommandables et nous les avons analysés. Quand nous avons cru que l'auteur n'était pas clair, ou trop long ou trop savant, suivant les circonstances nous y avons substitué 
notre texte, nous avons modifié le sien, nous y avons ajouté ou retranché; et quand nous avons cru que ne pouvions mieux faire ou que nous eussions fait pire, nous avons copié le texte pur et simple de l'auteur, comme on pourra le voir en plusieurs endroits. ${ }^{30}$

Cauchon destine donc son traité à un large public (écoles élémentaires et collèges classiques). Puisqu'il s'inspire fortement d'auteurs spécifiques pour les bases de la physique qu'il présente, il sera possible de retrouver des similitudes avec les quelques ouvrages lui ayant servi de référence.

Avant de s'attarder au détail des emprunts, il importe d'abord de repérer la source qui a inspiré le plan général des Notions élémentaires. Nous avons déjà noté que le cours de sciences du Séminaire de Québec était partiellement fondé sur le traité de physique de Péclet. Il s'agit d'un point de départ logique pour notre enquête. Le tableau 1 contient les plans respectifs des manuels de Cauchon et de Péclet (fort semblables à première vue).

Tableau 1

Plans comparés des manuels de physique de Péclet et de Cauchon

\begin{tabular}{|l|l|}
\hline Cauchon, $\mathbf{1 8 4 1 .}$ & Péclet, $\mathbf{1 8 3 8 .}$ \\
\hline Corps Pondérables & Corps Pondérables \\
Propriétés générales des corps & Propriétés générales des corps \\
Corps liquides & Propriétés des corps solides \\
Corps gazeux & Propriétés des corps liquides \\
& $\begin{array}{l}\text { Propriétés des corps gazeux } \\
\text { Acoustique }\end{array}$ \\
\hline $\begin{array}{l}\text { Fluides impondérables } \\
\text { Du calorique } \\
\text { De l'électricité } \\
\text { De l'électricité galvanique } \\
\text { Magnétisme } \\
\text { Lumière }\end{array}$ & $\begin{array}{l}\text { Fluides impondérables } \\
\text { Météorologie }\end{array}$ \\
\hline
\end{tabular}

La section sur les corps pondérables traite des propriétés et des lois de la mécanique qui s'appliquent aux objets possédant une masse. Cette portion de la physique regroupe la plupart des thèmes déjà bien établis au début du $19^{\mathrm{e}}$ siècle. La partie sur les fluides impondérables aborde plutôt les phénomènes impliquant un fluide sans masse. Récemment découverts ou faisant l'objet de débats, des sujets comme la chaleur, la lumière ou l'électricité sont au cœur des travaux de plusieurs physiciens de l'époque.

Au-delà des ressemblances entre les plans, les contenus de certaines sections de Cauchon et de Péclet sont plutôt analogues. À titre d'exemple, citons un extrait qui porte sur la propriété qu'ont les corps de se diviser (tabl. 2). 
Tableau 2

Extraits comparés des manuels de physique de Péclet et de Cauchon

\begin{tabular}{|c|c|}
\hline Cauchon, 1841, p. 7 & Péclet, 1838, tome 1, p. 8 \\
\hline $\begin{array}{l}<<\text { Comme chacune de ces lames peut } \\
\text { être divisée dans le sens de sa largeur } \\
\text { en deux parties visibles, et que chaque } \\
\text { 9/20me à peu près de ligne en longueur } \\
\text { peut être également divisé en huit } \\
\text { parties appréciables, on obtiendra par } \\
\text { cette opération } 14 \text { millions de parties } \\
\text { visibles }>>\text {. }\end{array}$ & $\begin{array}{l}<<\text { Chacune de ces lames pouvant être } \\
\text { divisée dans le sens de la largeur en deux } \\
\text { parties visibles, et chaque milimètre en } \\
\text { longueur pouvant également être divisé } \\
\text { en huit parties appréciables, on obtiendra, } \\
\text { par cette opération, quatorze billions de } \\
\text { parties visibles }>>\text {. }\end{array}$ \\
\hline
\end{tabular}

Des exemples similaires ont été identifiés dans les sections sur l'acoustique, le calorique, les lois du mouvement, l'électricité, l'optique et les corps gazeux, liquides et solides.

Malgré ces ressemblances, précisons toutefois que les Notions et le manuel de Péclet ne sont pas des copies conformes. Les sources de Cauchon sont multiples. Dans la section sur l'" emploi des corps solides pour transmettre et modifier les forces ", il s'inspire du Traité de Jérôme Demers. ${ }^{31}$ Dans une autre section sur l'« emploi de la vapeur comme force motrice ", il emprunte clairement des passages au manuel du Français César-Mansuète Despretz. ${ }^{32}$ Pour sa discussion sur les piles voltaïques et la météorologie, c'est au traité du Français Claude Pouillet ${ }^{33}$ que Cauchon accorde sa préférence. ${ }^{34}$ De plus, Cauchon s'inspire à l'occasion des nouvelles scientifiques du journal Le Canadien (au moment où il en assure la rédaction en 1840-1841). Afin de repérer ces emprunts, nous avons daté les découvertes et inventions qui paraissent les plus récentes pour la période considérée (fig. 2).

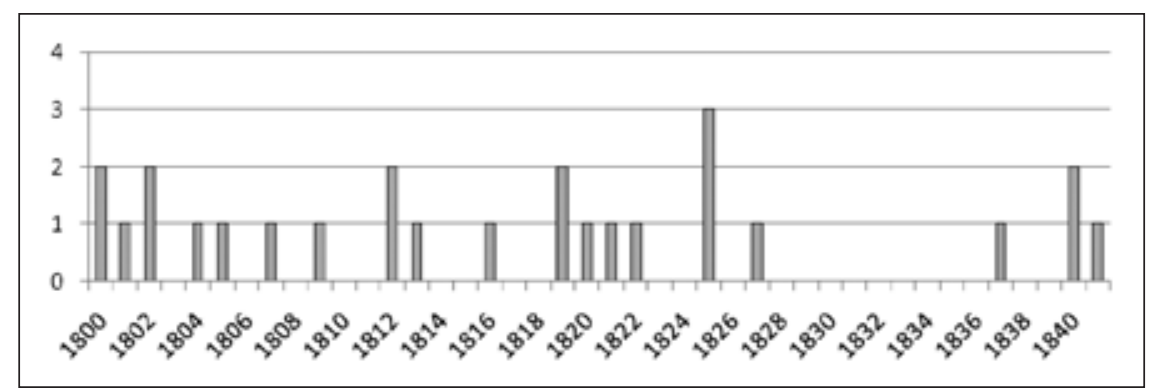

Figure 2 : Histogramme des découvertes et événements répertoriés dans les Notions

La figure 2 laisse voir deux blocs distincts séparés par une tranche d'une dizaine d'années. Avant 1827, les événements compilés se retrouvent dans les manuels dont il a déjà été question. Ainsi, la mesure de la vitesse du son dans l'eau par Colladon (1827), ${ }^{35}$ le perfectionnement de la chambre obscure par Chevalier $(1825)^{36}$ et l'invention de l'électroaimant par Arago (1821) ${ }^{37}$ figurent dans le traité de Péclet. En 
contrepartie, les événements qui se déroulent après 1837 n'apparaissent pas dans les manuels consultés et proviennent du journal Le Canadien. À titre d'exemple, mentionnons l'annonce du forage du puits de Grenelles à Paris $(1840)^{38}$ et le compte rendu d'une expérience "électrique " menée sur un pendu aux États-Unis (1841). ${ }^{39}$

Pour compléter notre discussion sur l'origine des contenus, notons que Cauchon inclut aussi des passages de sa composition qui font référence à des phénomènes locaux :

Les liquides et les solides jouissent aussi de cette propriété de transmettre le son. [...] C'est ainsi que pendant le siège de Québec, en mettant l'oreille contre terre, les habitants de Varennes entendaient le bruit du canon, à plus de 50 lieues de distance. ${ }^{40}$

Outre ce genre d'extraits facilement repérables en raison des indications spatio-temporelles, il est possible d'identifier certains passages des Notions qui ne semblent pas tirés des sources principales analysées auparavant. On peut penser qu'ils viennent de Cauchon lui-même. Soulignons toutefois que la majorité de ces passages ne concerne pas des points de théorie, mais relève plutôt de l'application, de l'illustration ou de l'anecdote.

\section{Contenu comparé du manuel de Cauchon et de manuels français et américains}

Pour les fins de notre étude comparative, les manuels considérés sont donc ceux destinés aux collèges royaux français et aux high schools américains, filières d'enseignement ressemblant le plus aux collèges classiques bas-canadiens. Les quatre ouvrages français de notre échantillon respectent le programme édicté pour l'enseignement de la physique dans les collèges royaux et sont approuvés par le Conseil royal de l'Instruction publique. ${ }^{41}$ Trois de ces manuels, ceux de Péclet, Despretz et Pouillet, ont servi de source d'inspiration à Cauchon pour la rédaction des Notions. ${ }^{42}$ Du côté américain, les manuels choisis sont ceux de J. L. Comstock et de Denison Olmsted, tous deux destinés aux "schools and academies $"{ }^{43}$ Leur tirage important et leurs nombreuses rééditions au fil du temps en font des points de référence pertinents. ${ }^{44}$ Nous utiliserons également les résultats de l'étude de Nietz menée sur plus d'une dizaine de manuels américains publiés entre 1826 et $1843 .{ }^{45}$

Le Tableau 3 présente l'importance accordée par chaque auteur aux différentes parties de la physique. Il s'agit en fait d'une proportion calculée selon le nombre de pages réservées à huit grands thèmes de la physique.

Précisons tout d'abord qu'il est ici question d'importance relative, indiquant les priorités des auteurs en faisant abstraction du nombre absolu de pages (très différent d'un manuel à l'autre). À titre d'exemple, si 22,9\% des pages des Notions sont consacrées à la mécanique, comparativement à 12,3\% chez Pouillet, il demeure que l'auteur français consacre 220 pages au sujet comparativement à 28 pour Cauchon. Il y a d'ailleurs une différence marquée entre l'étendue moyenne des manuels français 


\section{Tableau 3 \\ Espace consacré aux principaux thèmes dans les Notions élémentaires de physique et les manuels français et américains}

\begin{tabular}{|c|c|c|c|c|c|c|c|c|c|c|c|c|c|}
\hline Nationalité & Année & Auteur & $\begin{array}{l}0 \\
0 \\
0 \\
\mathbb{0} \\
0 \\
0 \\
2 \\
Z\end{array}$ & 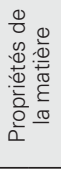 & 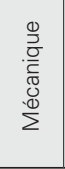 & 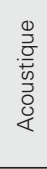 & $\begin{array}{l}\frac{亏}{J} \\
\frac{D}{\mathbb{D}} \\
\frac{\mathcal{U}}{U}\end{array}$ & 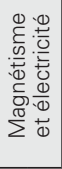 & $\frac{\stackrel{0}{\partial}}{\frac{0}{0}}$ & 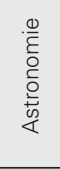 & 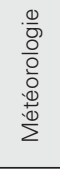 & 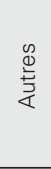 & 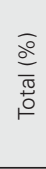 \\
\hline Français & 1836 & Despretz & 906 & 4,3 & 14,5 & 6,2 & 20,3 & 24,7 & 21,3 & 0 & 8,7 & 0 & 100 \\
\hline Français & 1839 & Deguin & 823 & 3,5 & 17,4 & 5,5 & 20,0 & 27,1 & 19,0 & 0 & 6,9 & 0,6 & 100 \\
\hline Français & 1832 & Pouillet & 1795 & 7,6 & 12,3 & 6,5 & 16,7 & 22,0 & 24,7 & 0 & 9,6 & 0,5 & 100 \\
\hline Français & 1838 & Péclet & 1179 & 3,7 & 18,2 & 6,1 & 18,9 & 22,2 & 24,8 & 0 & 6,0 & 0 & 100 \\
\hline \multicolumn{3}{|c|}{ Moyenne (Français) } & 1176 & 4,8 & 15,6 & 6,1 & 19,0 & 24,0 & 22,5 & 0 & 7,8 & 0,3 & 100 \\
\hline Américain & 1837 & Olmsted & 340 & 3,2 & 47,1 & 4,1 & 2,6 & 18,5 & 19,1 & 0 & 5,0 & 0,3 & 100 \\
\hline Américain & 1840 & Comstock & 359 & 3,0 & 37,4 & 2,1 & 4,8 & 11,4 & 16,5 & 22,8 & 1,8 & 0,3 & 100 \\
\hline Américain & $1826-43$ & Étude Nietz & $343^{*}$ & 4,5 & 37,9 & 2,6 & 9,3 & 14,4 & 19,3 & 11,7 & $* *$ & 0,3 & 100 \\
\hline \multicolumn{3}{|c|}{ Moyenne (Américains) } & 347 & 3,6 & 40,8 & 2,9 & 5,6 & 14,8 & 18,3 & 11,5 & 3,4 & 0,3 & 100 \\
\hline Canadien & 1841 & Cauchon & 124 & 7,6 & 22,9 & 9,3 & 15,3 & 16,1 & 14,4 & 0 & 12,7 & 1,7 & 100 \\
\hline
\end{tabular}

\begin{tabular}{|l|c|c|c|c|c|c|c|c|c|c|}
\hline Notions vs manuels français & 2,8 & 7,3 & 3,2 & $-3,7$ & $-7,9$ & $-8,1$ & 0 & 4,9 & 1,4 & 0,81 \\
\hline Notions vs manuels américains & 4,0 & $-17,9$ & 6,4 & 9,7 & 1,3 & $-3,9$ & $-11,5$ & 9,3 & 1,4 & 0,67 \\
\hline Manuels américains vs français & $-1,2$ & 25,2 & $-3,1$ & $-13,4$ & $-9,2$ & $-4,1$ & 11,5 & $-4,4$ & 0 & 0,47 \\
\hline
\end{tabular}

* Moyenne pour les 12 manuels considérés par Nietz.

* Catégorie absente dans l'étude de Nietz.

** Facteur de corrélation calculé selon les écarts (0 à 1).

(1176 p.), celle des manuels américains (347 p.) et celle des Notions (124 p.). Ceci étant dit, on remarque dans le Tableau 3 que le traité de Cauchon semble s'apparenter davantage aux manuels français (facteur de corrélation de 0,81) qu'aux manuels américains (facteur de corrélation de 0,67 ). Cette constatation n'est pas surprenante si on se rappelle que les Notions sont inspirées en partie des traités français de Péclet, Pouillet et Despretz.

\section{Les corps pondérables et la météorologie}

Contrairement à ses sources françaises, Cauchon accorde toutefois plus d'importance aux corps pondérables et aux thèmes concrets de la physique (propriétés de la matière, mécanique, acoustique, météorologie) qu'aux thèmes plus abstraits des fluides impondérables (chaleur, optique, électricité et magnétisme). En proportion, le jeune auteur canadien réserve $54 \%$ des pages de son traité aux fluides pondérables et à la météorologie comparativement à $35 \%$ pour les Français, soit un écart de près de $20 \%$. Dans les Notions, le contenu des sections sur la mécanique confirme par ailleurs la prédilection de Cauchon pour les connaissances simples et appliquées. Sur les 27 
pages du manuel traitant de mécanique, 5 sont réservées au levier, à la poulie, au coin et au plan incliné (19\% de la section). Chez Péclet et Nicolas Deguin, ${ }^{46}$ seulement $1,3 \%$ (3 sur 215 ) et $1,4 \%$ (2 sur 143 ) des pages sur la mécanique traitent des machines simples. En ce qui a trait à l'acoustique et à la météorologie, plusieurs sujets abordés par Cauchon sont aussi d'intérêt commun tels la pluie, la musique, l'ouie, le vent, le brouillard, les nuages, la voix, etc. Dans l'ensemble, sa présentation est donc plutôt descriptive et empirique.

Malgré un découpage différent, les manuels américains contiennent eux aussi une bonne quantité de connaissances appliquées. Toutes proportions gardées, la mécanique occupe $25 \%$ plus d'espace dans les traités de physique américains que dans les traités français. Aux États-Unis, les auteurs ont d'ailleurs coutume de réserver un chapitre distinct à l'hydrostatique, à l'hydraulique, à la pneumatique et aux lois de la gravité, ${ }^{47}$ contrairement à leurs homologues français qui regroupent ces sujets dans une même section ou un même chapitre. De plus, les ouvrages de Comstock et d'Olmsted expliquent en détails les nombreuses applications des machines simples (engrenages, treuils), de l'hydrostatique (presses, aqueducs), de l'hydraulique (moulins) et de la pneumatique (pompes à air, pistons), se démarquant ainsi des manuels français davantage axés sur la démonstration des lois du mouvement et de la gravité. ${ }^{48}$

\section{L'optique}

Dans la section sur l'optique, Cauchon énonce deux conceptions distinctes de la lumière, soit comme une onde (Descartes) ou comme un flot de particules (Newton). Il explique ensuite certaines propriétés élémentaires des faisceaux lumineux pour rapidement enchaîner avec l'explication de la vision et la description des principaux instruments d'optique. En accord avec son souhait de présenter "les phénomènes les plus ordinaires de la nature ", ${ }^{49}$ il réserve quatre pages au simple phénomène de l'arc-en-ciel. Dans les manuels français, la proportion théorie/application est d'un ordre égal ou supérieur à 3 pour 1 . Péclet, tout comme Deguin, discute longuement de réfraction, de polarisation et du système des ondulations, appuyé en cela par les découvertes récentes de Thomas Young et d'Augustin-Jean Fresnel. ${ }^{50}$ Chez Cauchon, pas un mot sur les nouvelles expériences appuyant la théorie ondulatoire, et seulement quelques lignes sur la polarisation et la diffraction.

L'optique occupe une proportion sensiblement équivalente des manuels français et américains. À l'intérieur de ce thème, il y a toutefois quelques différences de priorité entre les auteurs (tabl. 4).

Comstock et Olmsted semblent prioriser les phénomènes de réflexion et de réfraction au détriment de la diffraction et de la polarisation. On n'y trouve aucune remarque sur l'interférence et les expériences de Young et Fresnel, ou même sur les théories concurrentes ondes/particules des faisceaux lumineux. Aux discussions théoriques sur la nature de la lumière, Comstock et Olmsted privilégient plutôt l'explication des miroirs (réflexion), des lentilles (réfraction) et des instruments d'optique (microscope et télescope). 


\section{Tableau 4 \\ Espace consacré aux sous-thèmes de l'optique dans certains manuels français et américains}

\begin{tabular}{|l|c|c|c|c|c|c|}
\cline { 2 - 7 } \multicolumn{1}{c|}{} & \multicolumn{3}{c|}{ Américains } & \multicolumn{3}{c|}{ Français } \\
\cline { 2 - 8 } \multicolumn{1}{c|}{} & Comstock & Olmsted & Moyenne & Péclet & Despretz & Moyenne \\
\hline Nature, vitesse et caract. de la lumière & 5.0 & 4.1 & 4.6 & 12.2 & 6.9 & 9.6 \\
Réflexion, réfraction et spectre & 58.3 & 38.4 & 48.3 & 31.3 & 35.6 & 33.5 \\
Diffraction et polarisation & 0.0 & 0.0 & 0.0 & 40.5 & 30.9 & 35.7 \\
Vision & 15.0 & 15.1 & 15.0 & 4.1 & 6.9 & 5.5 \\
Instruments d'optique & 21.7 & 42.5 & 32.1 & 10.2 & 18.6 & 14.4 \\
Autres & 0.0 & 0.0 & 0.0 & 1.7 & 1.1 & 1.4 \\
\hline Total & 100 & 100 & 100 & 100 & 100 & 100 \\
\hline
\end{tabular}

\section{La chaleur}

Cauchon présente la théorie du calorique qui pose l'existence d'un fluide s'écoulant des corps chauds vers les corps froids. Il en distingue les formes sensibles et latentes. Les auteurs français consultés adoptent également cette théorie qui est la plus répandue à l'époque. Dans l'ensemble, ils accordent toutefois davantage d'espace à des considérations théoriques sur la dilatation et les changements de phase.

Chez Comstock et Olmsted, les discussions sur la chaleur sont limitées aux applications de la force motrice de la vapeur (machine de Watt, locomotive, bateau à vapeur, etc.) comptant pour un maigre 3,7 \% de leur traité. Contrairement à leurs homologues français, ces auteurs ne font aucune remarque sur le calorique, la dilatation ou les changements de phase. Les deux manuels américains sont d'ailleurs dépourvus d'un chapitre spécifiquement consacré à la chaleur, ce qui les distingue des traités français où cette matière est traitée séparément et occupe en moyenne 19 $\%$ de l'espace.

\section{Le magnétisme et l'électricité}

Cauchon accorde encore une fois une grande importance à la description d'applications et d'appareils (boussole, lumière électrique, pile, etc.). Sur le plan théorique, il distingue l'électricité vitreuse et l'électricité résineuse, en plus d'énoncer la loi de Coulomb sur les attractions et les répulsions électriques. Il discute en outre très brièvement des phénomènes électromagnétiques nouvellement découverts. Pour le magnétisme, il énonce essentiellement les caractéristiques générales de l'aimantation et du magnétisme terrestre. Chez les auteurs français consultés, les rapports entre les courants et les aimants sont étudiés plus en profondeur. Ainsi, Péclet et Deguin consacrent respectivement 53 et 40 pages de leur étude sur les fluides électriques aux phénomènes de l'électromagnétisme. En comparaison des Notions, les manuels français présentent également un ratio théorie/application plus élevé. 
Les données chiffrées de Nietz sur l'électricité et le magnétisme indiquent que les auteurs américains sont légèrement plus succincts que leurs homologues français. Il a toutefois été difficile de cerner cette tendance dans les deux manuels que nous avons étudiés. Olmsted accorde beaucoup d'importance aux manifestations des fluides électriques : effets mécaniques, chimiques et physiologiques; déviation des aiguilles de la boussole; machines électriques, etc. Il présente également les principes fondamentaux de la répulsion, de l'attraction et de la conduction, mais il s'abstient d'aborder l'électromagnétisme, découvert en 1820 par Christian Oersted. Si Comstock accorde moins d'espace qu'Olmsted à l'électricité et au magnétisme, il semble néanmoins très intéressé par l'électromagnétisme. Il y consacre 16 pages dans lesquelles il discute entre autres des solénoïdes, des électro-aimants et de la manière de produire des révolutions avec un courant et un aimant.

\section{L'astronomie}

Cauchon ne discute pas des phénomènes célestes. Les auteurs français étudiés non plus, car cette matière est enseignée séparément à l'aide de manuels d'astronomie. Du côté américain, elle ne figure pas dans tous les traités. Selon Nietz, " nine of the fourteen [textbooks] published before 1845 dealt with astronomy ". ${ }^{51}$ Comstock, qui y consacre près du quart de son traité, aborde des sujets allant des comètes aux constellations, en passant par le mouvement des planètes et l'explication des marées. Olmsted, en contrepartie, élague complètement le sujet.

\section{Forme et éléments didactiques des manuels}

Au-delà du contenu, les manuels scolaires sont d'abord et avant tout des aides pour faciliter l'apprentissage des élèves. Au début du $19^{\mathrm{e}}$ siècle, les auteurs de traités de physique commencent progressivement à manifester un souci pour la didactique (de manière toutefois inégale). À la lecture des travaux de Nietz et de Balpe sur les manuels de sciences, nous avons cerné dix éléments didactiques pertinents pour les besoins de notre étude comparative (tabl. 5). ${ }^{52}$

Les deux premiers éléments qui ont capté notre attention sont la présence de questions et de résumés destinés à confirmer la matière du cours. Les Notions en sont dépourvues. Contrairement à leurs homologues français, les auteurs américains semblent plus enclins à questionner leurs lecteurs. En 1845, Walter Johnson précise d'ailleurs dans la préface de son traité : "The general practice of introducing into class-books questions for examination is so well established as to need little comment $" .{ }^{53}$ Les manuels américains consultés renferment en moyenne deux ou trois questions par page. Celles-ci récapitulent les points importants du corps de texte, tout en suscitant la réflexion chez l'élève. Certaines questions sont qualitatives et font appel à la mémoire ou à la compréhension de l'élève; d'autres (plus rares) sont quantitatives et demandent l'application de formules. En ce qui concerne la présence de résumés, la pratique ne semble pas populaire d'un côté ou l'autre de l'Atlantique. Seul Comstock fournit quelques synthèses de ses chapitres. 
Tableau 5

Éléments didactiques dans les Notions et les manuels français et américains

\begin{tabular}{|c|c|c|c|c|c|c|c|c|c|}
\hline & 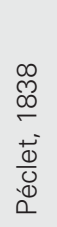 & 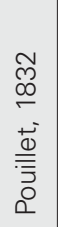 & 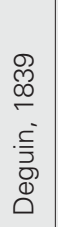 & $\begin{array}{l}0 \\
\infty \\
\infty \\
\circ \\
N \\
N \\
0 \\
0 \\
0 \\
0 \\
0\end{array}$ & 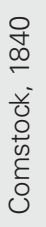 & 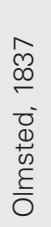 & 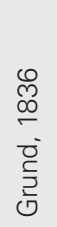 & 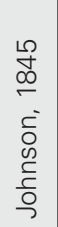 & 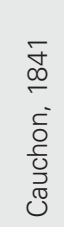 \\
\hline Présence de questions & 0 & 0 & 0 & 0 & 1 & 1 & 1 & 1 & 0 \\
\hline Présence de résumés & 0 & 0 & 0 & 0 & 1 & 0 & 0 & 0 & 0 \\
\hline Numérotation des sections & 1 & 1 & 1 & 1 & 1 & 1 & 1 & 1 & 0 \\
\hline Sous-titres de sections & 1 & 0 & 1 & 1 & 1 & 1 & 1 & 1 & 1 \\
\hline Présence d'un index & 0 & 0 & 0 & 0 & 1 & 0 & 0 & 1 & 0 \\
\hline Présence de notes de bas de page & 1 & 1 & 0 & 1 & 0 & 1 & 1 & 1 & 0 \\
\hline Portions de texte en retrait ou itallique & 1 & 0 & 0 & 0 & 0 & 1 & 1 & 0 & 0 \\
\hline Figures intégrées au texte & 0 & 0 & 0 & 0 & 1 & 1 & 1 & 1 & 0 \\
\hline Nombres arrondis pour exemples & 0 & 1 & 1 & 0 & 1 & 1 & 1 & 1 & 1 \\
\hline Recommandations au professeur & 0 & 0 & 0 & 0 & 1 & 1 & 1 & 1 & 0 \\
\hline Total & 4 & 3 & 3 & 3 & 8 & 8 & 8 & 8 & 2 \\
\hline Moyenne & \multicolumn{4}{|c|}{$3,3 / 10$} & \multicolumn{4}{|c|}{$8 / 10$} & $2 / 10$ \\
\hline
\end{tabular}

Les numéros et les sous-titres identifiant les sections et les sous-sections sont des éléments didactiques facilitant le repérage de l'information. Ces marqueurs sont plutôt communs dans les manuels de sciences du début du $19^{\mathrm{e}}$ siècle. Si Cauchon ne numérote pas ses sections, il prend toutefois soin de placer en italique le mot résumant le mieux le contenu de chaque paragraphe. Moins populaire, l'index alphabétique facilite également la localisation rapide d'informations. Seuls les traités de Comstock et de Johnson comportent un index. Soulignons cependant que la plupart des manuels étudiés, y compris les Notions, contiennent tout de même une table des matières.

Les notes de bas de page et les portions de texte en retrait ou en italique (trois lignes et plus) permettent de séparer l'information essentielle de celle qui est complémentaire. Plus précisément, elles servent : 1) à séparer les démonstrations, les exemples chiffrés et les récits d'expérience du texte principal; 2) à mettre en évidence l'énoncé de lois ou de propriétés fondamentales; 3) à apporter une précision, en évitant d'alourdir inutilement le corps principal du texte. Ces trois usages sont assez répandus chez les auteurs consultés.

Le huitième élément du tableau 5, l'intégration des figures au texte, semble peu important à première vue. Joindre l'image au mot comporte toutefois l'avantage non négligeable de limiter l'effort devant être accompli par l'élève. Chez les auteurs américains consultés, les figures sont avantageusement imbriquées dans le corps du texte, ce qui les rend plus accessibles au lecteur (fig. 3). Dans les Notions, tout comme dans les traités français, les figures sont regroupées sur des planches pliables placées à la fin 
de l'ouvrage. Il n'est pas certain que l'élève se donne toujours la peine de retourner à la fin du manuel pour consulter l'image appuyant la théorie.

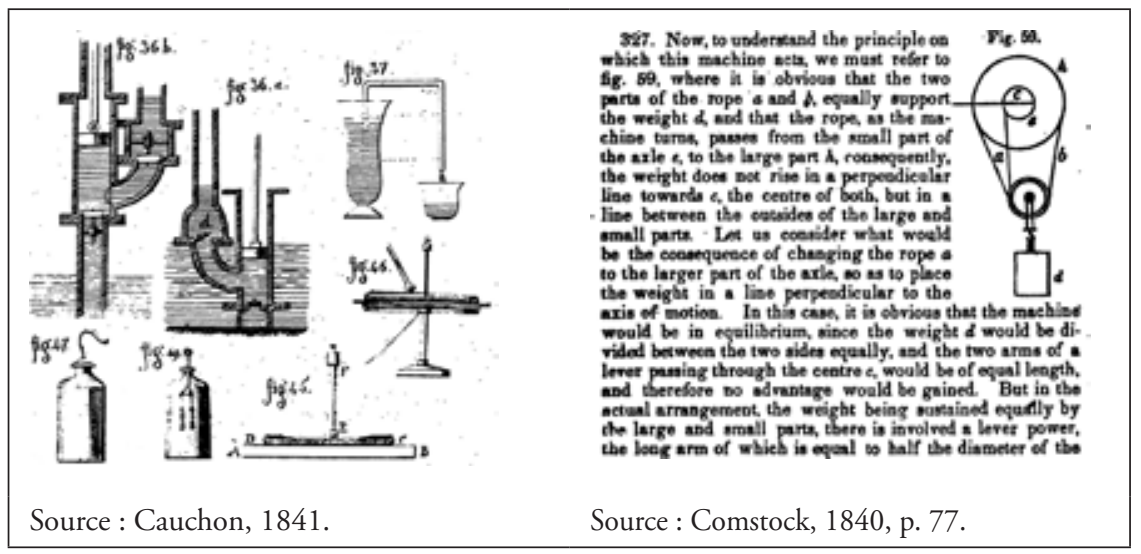

Figure 3 : Exemples de figures dans les manuels de physique de Cauchon et de Comstock.

L'utilisation de nombres arrondis pour les exemples et les démonstrations est un élément didactique tout aussi subtil que le précédent. Bien que ce soit souvent par souci de précision, le choix de faire suivre les décimales nuit généralement à la valeur pédagogique d'un exposé. À titre d'exemple, Péclet indique qu'en chute libre, un objet parcourt 4,9044 mètres dans la première seconde. Comstock arrondit plutôt cette mesure à 16 pieds $(4,87 \mathrm{~m})$. Avec cette approximation, il peut, contrairement à Péclet, conserver des nombres entiers pour ses calculs, illustrant l'accélération des corps. Ainsi employés, les chiffres arrondis ou approximés constituent un élément didactique positif.

Le dernier aspect des manuels méritant notre attention est la présence de recommandations adressées aux maîtres ou aux lecteurs. Celles-ci peuvent être de natures multiples. Olmsted suggère une liste d'instruments scientifiques indispensables pour donner un cours de physique digne de ce nom. ${ }^{54}$ Comstock souligne les sections de son manuel que l'étudiant doit prioritairement assimiler. ${ }^{55}$ Johnson recommande à différentes occasions la lecture de traités américains, britanniques et français pouvant avantageusement compléter sa présentation. ${ }^{56}$ Dans l'ensemble, les commentaires renforcent la valeur pédagogique des ouvrages et permettent d'établir un dialogue avec le lecteur. Encore une fois, seuls les manuels américains consultés renferment de telles recommandations. Cauchon et les auteurs français ne s'adressent pas directement au professeur ou à l'étudiant.

\section{Manuels français : encyclopédisme et exhaustivité}

En France, nous avons constaté que les manuels de physique employés dans les collèges royaux sont très volumineux (généralement plus de 800 pages). Les auteurs accordent beaucoup d'importance aux thèmes plutôt abstraits des fluides impondérables 
(encore en développement à l'époque), au détriment de la mécanique et des thèmes plus appliqués de la physique. Ils développent avec moult détails leur exposé et sont fervents de précision, avec une propension pour les décimales.

Pour expliquer cette tendance à un certain encyclopédisme dans les traités de physique français, il est d'abord intéressant de se pencher sur le statut de leur auteur. Ce sont pour la plupart des scientifiques. Péclet figure parmi les fondateurs de l'École centrale des Arts et Manufactures, ce qui lui vaut d'être considéré comme l'" un des créateurs de la physique industrielle $» .^{57}$ Pouillet est successivement suppléant, professeur adjoint et professeur titulaire à la Faculté des sciences de Paris. Il rédige entre autres pour les Annales de chimie et de physique un mémoire portant sur " l'électricité des fluides élastiques ». ${ }^{58}$ Despretz, selon une notice nécrologique, a " poursuivi, pendant plus de quarante ans, l'étude des grands phénomènes de la physique : le son, l'électricité, la densité des liquides, etc. ".59

C'est donc avec un bagage scientifique assez élaboré que les auteurs français considérés entreprennent la rédaction de leur manuel de physique. Malgré une volonté louable d'introduire l'expérience dans l'enseignement de cette science, de permettre aux élèves de "voir et toucher ", ${ }^{60}$ c'est souvent l'habitus du savant qui transparaît le plus dans ces manuels de la première moitié du $19^{\mathrm{e}}$ siècle : expériences détaillées à outrance, discussions sur l'intérêt de recherches nouvelles, longues descriptions de montages expérimentaux, etc. Comme le précise Claudette Balpe dans son étude, lorsque les auteurs de manuels scolaires sont des savants ou des professeurs de l'enseignement supérieur, le niveau des ouvrages dépasse très souvent celui d'une classe de lycée. ${ }^{61}$

Les activités de recherche des auteurs doivent également être pris en compte pour comprendre la prépondérance de certains thèmes dans les traités de physique français. D'après les sujets privilégiés par Péclet, Pouillet et Despretz dans leurs recherches personnelles (chaleur, fluides électriques et optique) et les thèmes populaires dans les travaux des physiciens français influents de l'époque (Dulong sur la chaleur spécifique et la dilatation, Arago sur la tension de la vapeur et l'optique, Biot sur le magnétisme et la lumière polarisée, par exemple), il est aisé de comprendre l'engouement relatif pour les fluides impondérables dans les manuels de physique. Certains auteurs font d'ailleurs référence dans leur traité à leurs propres travaux de recherche, outrepassant largement le niveau de connaissances utiles pour un élève de collège. ${ }^{62}$

Dans un autre ordre d'idées, les manuels français semblent partager entre eux une structure et un contenu relativement similaires. On peut supposer que cette apparente uniformité s'explique dans un premier temps par la présence d'un niveau d'enseignement supérieur. En effet, la nécessité de préparer des élèves au concours d'institutions telles l'École polytechnique pousse les institutions de niveau secondaire à adapter les contenus enseignés. La tendance à l'uniformisation est en outre appuyée par les autorités scolaires. Selon un arrêté de 1821, «le Conseil royal fait publier, à la fin de chaque année scolaire, le catalogue des ouvrages dont les professeurs doivent se servir l'année suivante ". ${ }^{63}$

Notre étude a mis en évidence la faiblesse des traités de physique français sur le plan didactique. Les auteurs étant pour la plupart des scientifiques, et non des pédagogues, 
l'exactitude des connaissances prime généralement sur la limpidité de l'exposé. Renforçant cette tendance, l'école normale sélectionne et forme les futurs enseignants de lycée en fonction de leur érudition, et non de leurs aptitudes pédagogiques. ${ }^{64}$ Il en résulte un enseignement plutôt aride, laissant peu d'espace aux démonstrations et à la composante expérimentale. Avant l'introduction d'un cours de manipulation à l'école normale en 1837 , les compétences expérimentales des professeurs sont très inégales, d'où la faveur généralisée pour l'exposé magistral. ${ }^{65}$

\section{Manuels américains : pragmatisme et didactique}

Aux États-Unis, dans la première moitié du $19^{\mathrm{e}}$ siècle, les manuels de physique employés dans les high schools et les académies sont plutôt succincts, deux fois moins volumineux que les manuels français. Les auteurs prisent les aspects appliqués et pratiques de la physique (mécanique, machine à vapeur, appareils électriques, etc.), au détriment des connaissances plus abstraites. Afin de rendre le contenu accessible aux élèves et faciliter le travail de l'enseignant, la plupart des manuels sont assez bien conçus sur le plan didactique et sont écrits dans un style visant à établir un dialogue avec le lecteur.

Rappelons tout d'abord que le mouvement des high schools s'inscrit dans une volonté de démocratiser l'éducation, de décloisonner un système élitiste où l'enseignement supérieur classique fait figure de monopole. ${ }^{66}$ Bien que le cursus des high schools préserve une composante humaniste, axée sur les vertus civiques, un changement s'opère toutefois dans les visées de la formation offerte. En plus du traditionnel programme préparant au collège, des cours ouvrant sur les affaires, le commerce et les manufactures sont offerts. Il n'est plus question de former uniquement des hommes de lettres. ${ }^{67}$ Pour les réformateurs de l'époque, les high schools représentent un investissement dans le capital humain, car avec l'accroissement et la diversification des compétences, vient une augmentation de la productivité et de la richesse du pays. Dans les manuels de physique, cette volonté d'offrir à la jeunesse des connaissances pratiques, plus adaptées au marché du travail, est clairement affirmée par les choix de sujets. À titre d'exemple, Comstock discute longuement des machines simples et complexes, de l'hydraulique, de la force motrice de la vapeur et de l'électricité et précise qu'une bonne connaissance des lois du mouvement et des principes de la mécanique permet de construire des machines en prévenant " the loss of labour and money ". ${ }^{68}$

La facture générale des traités de physique américains semble également tributaire d'un certain pragmatisme hérité des auteurs britanniques, influencés par les rapides progrès de l'industrialisation. Les succès enregistrés dans les arts utiles témoignent d'une maîtrise concrète des sphères appliquées de la science. Les grammar schools britanniques, en plus des humanités, offrent des cours de sciences reposant sur des manuels dont certains s'illustrent par leur accessibilité. Ils serviront d'inspiration pour plusieurs auteurs américains. Mentionnons deux ouvrages britanniques, An Easy Grammar of Natural and Experimental Philosophy de Richard Philipps ${ }^{69}$ et Conversations on Natural Philosophy de Jane Marcet ${ }^{70}$, qui connaissent un succès indéniable aux États-Unis. Dans la première édition (1807) du traité de Philipps, 
on retrouve déjà 10 planches de dessins, 31 pages de questions, un glossaire-index de 12 pages et de nombreux exemples d'applications illustrant la matière. Dans Conversations on Natural Philosophy, rééditées sous différentes formes aux États-Unis, l'auteure établit un dialogue avec l'élève dans le but d'aborder les éléments appliqués de la physique que sont la puissance mécanique, l'hydraulique et la pneumatique. Sans déborder de notre cadre comparatif initial, mentionnons seulement qu'il existe des parallèles intéressants à établir entre les manuels britanniques et les manuels américains quant aux thèmes abordés et à la didactique.

Le contenu des manuels de physique témoigne en outre de la structure assez floue du système scolaire américain dans la première moitié du $19^{\mathrm{e}}$ siècle. Comme le mentionne Nietz, il est parfois ardu de différencier les manuels destinés aux high schools de ceux destinés aux collèges (universités) et aux classes supérieures des écoles élémentaires. À son avis, la raison de ce problème "was that most secondary schools were not carefully graded $»{ }^{71}$ Faute de directives communes à l'échelle nationale, les programmes scolaires sont très variables d'un État à l'autre, et parfois à l'intérieur même d'un État. Les high schools, coexistant avec les académies et les séminaires, fixent leur propre programme d'études et les enseignants sont libres d'adopter le manuel de leur préférence. Avant la création des écoles normales (la première est fondée en 1839), le choix du manuel est d'autant plus important que les maîtres enseignant à l'ordre secondaire ont souvent une formation ne dépassant pas le palier auquel ils enseignent. ${ }^{72}$ Leurs cours dépendent de la qualité des manuels et, par le fait même, du niveau de compétences de leur auteur. ${ }^{73}$

\section{Notions élémentaires de physique : un condensé pratique peu employé}

Le premier manuel de physique canadien, les Notions élémentaires de Cauchon, comporte un total de 124 pages, ce qui est nettement moins que les traités français et américains de l'époque. Comme on l'a vu, il est axé sur les thèmes concrets de la physique, et contient peu de formules et d'exemples chiffrés. Hormis la présence de planches de figures, la forme en est plutôt austère. Bien que l'exposé général des connaissances soit accessible et concis, l'auteur offre peu d'éléments pour renforcer la valeur didactique de sa présentation.

Rappelons tout d'abord que Cauchon est issu du cours classique qui constitue l'aboutissement du système scolaire bas-canadien dans la première moitié du $19^{\mathrm{e}}$ siècle. Contrairement à la plupart des auteurs de manuels français et américains, il n'est ni savant, ni professeur de science. En l'absence de véritables institutions d'enseignement supérieur, ces professions sont d'ailleurs pratiquement inexistantes au BasCanada. De là, un premier manuel de physique qui, bien qu'actuel, n'est pas très poussé sur le plan théorique et didactique. Destiné tant aux collèges qu'aux écoles élémentaires, le manuel de Cauchon vise un public très large, ce qui affaiblit probablement son utilité aux yeux de la plupart des professeurs. Au Séminaire de Québec, des enseignants tels Jérôme Demers et Louis-Jacques-Casault préferent construire leurs propres notes de cours en se basant sur des traités français destinés aux collèges 
royaux. Il en résulte des manuscrits plus élaborés que les Notions élémentaires de Cauchon. ${ }^{74}$

Sur l'édition, l'impression, la diffusion et la réception du manuel de Cauchon, nous avons très peu d'indications. L'éditeur, Jean-Baptiste Fréchette, est imprimeur puis copropriétaire du journal Le Canadien, d'où le lien avec Cauchon qui y travaille en $1841 .{ }^{75}$ Nous savons en outre qu'il est présent dans le domaine de l'édition scolaire et qu'on lui doit entre autres l'impression du Traité d'agriculture de Jean-François Perreault (1831), du Traité d'arithmétique de Joseph Laurin (1837) et de l'Essai de grammaire française d'Amable Berthelot (1840). ${ }^{76}$

Le traité de Cauchon a été vendu par souscription, bien que certaines copies se soient retrouvées en vente libre aux bureaux du Canadien et chez le libraire Fabre de Montréal. Nous pouvons estimer à quelques centaines le nombre de copies imprimées, tout au plus. Il semble qu'il n'ait jamais fait l'objet de réédition. À l'époque, les copies imprimées circulent probablement dans le cercle restreint de l'élite intellectuelle côtoyée par son auteur, mais leur pénétration en milieu scolaire est limitée ou du moins incertaine. Aujourd'hui, il en subsiste encore quelques exemplaires dans les bibliothèques universitaires et les fonds d'archives de collèges. ${ }^{77}$

\section{Conclusion}

Malgré leur diffusion restreinte au sein du système d'enseignement, le contenu des Notions élémentaires de physique rappelle l'importance que Cauchon accorde aux connaissances pratiques. Selon ses propres mots :

Combien de talents ingénieux et distingués pour la mécanique, pour la menuiserie, pour la charpente et pour tous les autres arts usuels, qui faute d'avoir des principes vrais et sûrs ne perceront jamais le voile qui couvre toutes ces belles facultés ? Avec ces connaissances [physiques] l'homme ne sera pas seulement une machine, une main d'œuvre, mais il se servira de sa pensée pour atteindre à une plus grande perfection. ${ }^{78}$

Ce discours fait écho aux préoccupations de l'époque en matière d'éducation. ${ }^{79} \mathrm{Au}$ Bas-Canada, un peu à l'image de ce qui a été observé aux États-Unis, les premières poussées de l'industrialisation et les besoins changeants du marché du travail induisent certaines modifications dans les visées du système scolaire. C'est dans cet esprit que des autodidactes, tel Cauchon en physique et Meilleur en chimie, ${ }^{80}$ produisent une première série de manuels de sciences présentant des connaissances adaptées aux besoins nouveaux du commerce et de l'industrie.

Si les Notions constituent un échec certain du point de vue de la pénétration en milieu scolaire, d'autres manuels de sciences pratiques bas-canadiens parus à la même époque connaissent un succès plus convaincant, comme le Traité d'agriculture de Perreault, qui est recommandé par le surintendant de l'Éducation pour l'usage des écoles et est réimprimé à deux reprises. ${ }^{81}$ L'infortune des Notions ne résulte donc pas de l'absence d'une demande pour des connaissances pratiques, mais plutôt du public 
trop large qu'elles visent : trop simples pour les collèges et probablement trop poussées pour les écoles élémentaires.

Par ailleurs, le traité de Cauchon permet de jauger la portée des connaissances scientifiques transmises dans les classes terminales du cours classique. Même si Cauchon s'inspire fortement d'auteurs français, c'est à ses professeurs du Séminaire de Québec qu'il emprunte en premier lieu. Malgré leur forme condensée, les Notions abordent la plupart des thèmes de la physique de l'époque. Le souci manifesté par Cauchon de mettre à jour le contenu de son manuel à l'aide de nouvelles tirées du Canadien confirme par ailleurs son intérêt et sa curiosité pour les sujets scientifiques. La première vague de traités de sciences paraissant à partir des années 1830 reflète un intérêt croissant pour les sciences, intérêt qui dépasse le contexte scolaire et qui touche de plus en plus différentes sphères de la société bas-canadienne.

Notre étude comparative du manuel de Cauchon avec les manuels français et américains a mis en lumière deux conceptions de l'enseignement de la physique : plus encyclopédique en France et plus pragmatique aux États-Unis. Elle a mis en relief les rapports entre contenu et style des manuels (connaissances appliquées versus connaissances abstraites, présence ou absence d'éléments didactiques), et les conditions institutionnelles et pédagogiques de leur utilisation (formation et occupation des auteurs, compétence des enseignants, démarcation entre les paliers d'enseignement). À notre avis, une telle approche permet d'éviter les évaluations simplistes de la portée et de l'utilité des manuels dans des contextes nationaux différents. Elle inscrit les manuels dans un cadre plus large en tant que reflets d'une réalité scolaire, sans pour autant leur attribuer un pouvoir intrinsèque de changement, d'innovation. Avant tout, il ne faut pas oublier que les livres d'écoles sont modelés par les contraintes du système scolaire et de la société dans laquelle ils circulent.

\section{Notes}

1 Joseph Cauchon, Notions élémentaires de physique (Québec: Fréchette, 1841).

2 John Herbert Sangster, Natural philosophy : part I, including statics, hydrostatics, pneumatics, dynamics, hydrodynamics, the general theory of undulations, the science of sound, the mechanical theory of music, etc. : designed for the use of normal and grammar schools and the higher classes in common schools (Montréal : John Lovell; Toronto, Robert \& Adam Miller, 1861).

3 Richard A. Jarrell, "The Influence of Irish Institutions upon the Organization and Diffusion of Science in Victorian Canada ", Scientia Canadensis 9, 2 (1985) : 157.

4 Paul Aubin et al., 300 ans de manuels scolaires au Québec (Montréal : BAnQ; Sainte-Foy : PUL, 2006), 13.

5 Claude Galarneau, «L'enseignement des sciences au Québec et Jérôme Demers (1765-1835) ", University of Ottawa Quarterly, section 1-2, vol. 47 (1977) : 84-94.

6 Luc Chartrand, Raymond Duchesne et Yves Gingras, Histoire des sciences au Québec, de la Nouvelle-France à nos jours (Montréal : Boréal, 2008), 218-219.

7 Claudette Balpe, Enseigner la physique au collège et au lycée, une approche historique (Rennes : Presses universitaires de Rennes, 2001).

8 John Alfred Nietz, The Evolution of American Secondary School Textbooks (Rutland: C. E. Tuttle, 1966). 
9 Andrée Désilets, "Cauchon, Joseph-Édouard ", Dictionnaire biographique du Canada en ligne (DBCL), http://www.biographi.ca/index-f.html.

10 Galarneau, "L'enseignement des sciences ", 84-94.

11 BAnQ, Séminaire de Québec, "Programme abrégé du cours d'études du Petit Séminaire de Québec, pour l'année 1838-9 », 2 août 1839. Les notes sont celles de Jérôme Demers, professeur de physique et de philosophie au Séminaire de Québec entre 1800 et 1835, ASQ, Manuscrit-15, Jérôme Demers, "Traité élémentaire de physique ", Québec, 1833. Le manuel français est celui d'Eugène Péclet, Traité élémentaire de physique, 2 tomes, $3^{\mathrm{e}}$ éd. (Paris : Hachette, 1838).

12 ASQ, Manuscrit-210, Pierre Boucher (suivant le cours de Casault), "Traité élémentaire de physique ", 1843-1844.

13 Pour plus de détails sur les cours de physique des collèges classiques bas-canadiens de la première moitié du $19^{\mathrm{e}}$ siècle, voir Alain Couillard, «Le premier manuel de physique publié au Québec : contexte et contenu des Notions élémentaires de physique de Joseph Cauchon " (mémoire de maîtrise, UQÀM, 2008), 41-64.

14 John Charles Dent, "The Hon. Joseph Edouard Cauchon », in The Canadian Portrait Gallery (Toronto : J.B. Magurn, 1881), vol. 4, 140.

15 Désilets, "Cauchon, Joseph-Édouard ", DBCL.

16 Pour plus de détails, consulter Alain Couillard, "Joseph Cauchon et le goût des sciences ", Cap-aux-Diamants, 92 (2008) : 21-24.

17 Napoléon Aubin, "Institut Vattemare », Le Fantasque, 26 février 1841, 150. Pour de plus amples détails sur ce projet d'institut, Couillard, «Le premier manuel de physique publié au Québec ", 21-25.

18 Micheline Cambron, dir., Le journal Le Canadien: Littérature, espace public et utopie, 1836-1845 (Montréal : Fides, 1999), 125-191.

19 Yvan Lamonde, Histoire sociale des idées au Québec, 1760-1896 (Montréal : Fides, 2000), 86.

20 Voir « Le goût des arts et des sciences au Bas-Canada " dans Chartrand, Duchesne et Gingras, Histoire des sciences au Québec, 75-108.

21 Lamonde, Histoire sociale des idées au Québec, 178.

22 Ibid., 408.

23 Ibid., 407.

24 Andrée Dufour, Histoire de l'éducation au Québec (Montréal : Boréal, 1997), 26.

25 Lamonde, Histoire sociale des idées, 87.

26 Charles-Elzéar Mondelet fait paraître une série de lettres dans The Canada Times en 1840 où il donne son avis sur les orientations que devrait prendre le système d'éducation au Bas-Canada. Appréciées par Lord Durham, elles seront plus tard publiées dans Letters on Elementary and Practical Education (Montréal : John James Williams, 1841), 6. Pour une étude des discours en ce sens, Robert Gagnon, "Les discours sur l'enseignement pratique au Canada français : 1850-1900 ", in Marcel Fournier, Yves Gingras et Othmar Keel, dir., Sciences et Médecine au Québec, perspectives sociohistoriques (Québec : IQRC, 1987), 19-36.

27 Lamonde, Histoire sociale des idées, 169.

28 Du point de vue des auteurs de manuels, l'argument économique pèse lourd dans la balance après l'adoption de l'Acte pour protéger la propriété littéraire en 1832. Cette nouvelle loi assure " les auteurs de publications littéraires, de gravures, de cartes ou d'œuvres de musique de même que leurs exécuteurs, administrateurs ou représentants légaux d'un droit de propriété de 28 ans à compter de la date de l'enregistrement de l'œuvre ", Yvan Lamonde, La librairie et l'édition à Montréal, 1776-1920, (Montréal : BAnQ, 1991), 44.

29 À titre d'exemple : Jean-Antoine Bouthillier, arpenteur et journaliste, Traité d'arithmétique pour l'usage des écoles (Québec: Neilson, 1809); Jean-Baptiste Meilleur, Cours abrégé de 
leçons de chymie (Montréal : Ludger Duvernay, 1833); Joseph Laurin, Traité d'arithmétique (Québec : Fréchette, 1837).

30 Joseph Cauchon, " Notions élémentaires de physique ", Le Canadien, 21 janvier 1842.

31 ASQ, M-15, Demers, "Traité élémentaire de physique ", articles 163 à 196.

32 César-Mansuète Despretz, Traité élémentaire de physique, $4^{\mathrm{e}}$ éd. (Paris : MéquignonMarvis, 1836).

33 Claude Pouillet, Éléments de physique expérimentale et de météorologie, 4 vol. (Paris : Béchet Jeune, 1827).

34 Sur le détail des emprunts, Couillard, « Le premier manuel de physique publié au Québec », 111-121.

35 Péclet, Traité élémentaire de physique, tome I, 280.

36 Ibid., tome II, 378-379.

37 Ibid., tome II, 203.

38 "Puits artésien de Grenelle ", Le Canadien, 5 avril 1841; "France ", Le Canadien, 16 avril 1841; "Académie Royale des Sciences, Séance du 1 ${ }^{\text {er }}$ mars ", Le Canadien, 30 avril 1841.

39 "Le pendu ressuscité ", Le Canadien, 7 mai 1841.

40 Cauchon, Notions, 42.

41 Balpe, Enseigner la physique, 63.

42 Le $4^{\mathrm{e}}$ manuel considéré est celui de Nicolas Deguin, Cours élémentaire de physique, $2^{\mathrm{e}}$ éd. (Toulouse : Martegoute, 1839).

43 J.L. Comstock, A System of Natural Philosophy, $53^{\mathrm{e}}$ éd. (New York : Robinson, 1840), 1; Denison Olmsted, A Compendium of Natural Philosophy (Charleston : Babcock, 1837), i.

44 Nietz estime le nombre de copies vendues à travers le monde des différentes éditions des manuels de Comstock et d'Olmsted à respectivement 600000 et 70000 (Nietz, The Evolution of American Secondary School Textbooks, 116-119.

45 Ibid., 144.

46 Nicolas Deguin, Cours élémentaire de physique.

47 Nietz, The Evolution of American Secondary School Textbooks, 130.

48 Certains chapitres d'Olmsted sont d'ailleurs uniquement réservés aux connaissances appliquées comme leur titre l'indique : "Projectiles and gunnery ", "Machinery " and "Mechanical agencies of air".

49 Cauchon, Notions, 4.

50 Thomas Young, The Bakerian Lecture : on the Theory of Light and Colours (London : W. Bulmer, 1802); Ausgustin-Jean Fresnel, " Mémoire sur la diffraction de la lumière ", Mémoires de l'Académie des Sciences 5 (1826) : 33-475.

51 Ibid., 131.

52 Pour étoffer notre comparaison, nous avons ajouté deux manuels américains destinés aux high schools: F.J. Grund, Elements of Natural Philosophy (Boston : Charles J. Hendee, 1836); W.R. Johnson, A System of Natural Philosophy (Philadelphia : Edward C. Biddle, 1845).

53 Johnson, A System of Natural Philosophy, 7.

54 Olmsted, A Compendium of Natural Philosophy, 355-359.

55 Comstock, A System of Natural Philosophy, 79, 323.

56 Johnson, A System of Natural Philosophy, 7-8.

57 Charles de Comberousse, "L'École Centrale des Arts et Manufactures ", La Nouvelle revue, $4^{e}$ année, tome 18 (1882) : 79.

58 Claude Pouillet, "Mémoire sur l'électricité des fluides élastiques, et sur une des causes de l'électricité de l'atmosphère ", Annales de chimie et de physique, tome 35 (1827) : 401-420

59 L. Figuier, L'année scientifique et industrielle, $8^{\mathrm{e}}$ année (Paris : Hachette, 1863), 536-538.

60 Claude Pouillet, Éléments de physique expérimentale et de météorologie (Paris : Béchet Jeune, 1832), tome 1 , v. 
61 Balpe, Enseigner la physique, 63.

62 À titre d'exemple, Péclet s'intéresse aux « raies que l'on observe à l'oeil nu à travers une fente étroite ", [s.n.], Annales de chimie et de physique, Table générale raisonnée des matières, 400. Dans son manuel, en commentaire des expériences de Fresnel, il précise " les effets résultant de l'interférence des ondes émanées de deux points devra se réaliser, si notre théorie est exacte », Traité élémentaire de physique (1832), tome 2, 508 (c'est nous qui soulignons).

63 [s.n.], Recueil des lois et des réglemens concernant l'instruction publique (Paris : BrunotLabbe, 1824), tome 7, 115.

64 Sur la formation des professeurs, voir l'introduction dans Philippe Savoie, Les enseignants du secondaire XIXe $-X X^{e}$ siècles, le corps, le métier, les carrières (Paris : Institut national de recherche pédagogique, 2000), tome 1, 15-87.

65 Nicole Hulin, L'enseignement secondaire scientifique en France d'un siècle à l'autre, 1802-1980 (Lyon : Institut national de recherche pédagogique, 2007), 71.

66 D.F. Labaree, The Making of an American High School, The Credentials Market and the Central High School of Philadelphia, 1838-1839 (New Haven : Yale University Press, 1988), 12-14.

67 Ibid., 19-23.

68 Comstock, A System of Natural Philosophy, 66.

69 Richard Philipps (alias David Blair), An Easy Grammar of Natural and Experimental Philosophy (London: W. Eliot, 1807).

70 Jane Marcet, Conversations on Natural Philosophy (London : Longman, 1819).

71 Ibid., 2.

72 Nietz, The Evolution of American Secondary School Textbooks, 6.

73 À titre indicatif, Olmsted et Johnson sont professeurs dans des institutions d'enseignement supérieur. Comstock et Grund sont spécialisés dans la rédaction d'ouvrages scolaires.

74 ASQ, Demers, M-15; ASQ, Casault, M-210.

75 Jean-Charles Falardeau, "Parent, Étienne ", Dictionnaire biographique du Canada en ligne (DBCL), http://www.biographi.ca/index-f.html.

76 Voir le site de Paul Aubin sur les manuels scolaires, www.bibl.ulaval.ca/ress/manscol, rubrique : dictionnaire des auteurs.

77 Le manuel de Cauchon ne figure pas dans le catalogue dressé par Paul Aubin et Michel Simard, Les manuels scolaires dans la correspondance du Département de l'instruction publique, 1842-1899: inventaire (Sherbrooke : Éditions Ex libris, 1997). Pour plus de détails sur la diffusion du manuel de Cauchon, voir Couillard, "Le premier manuel de physique publié au Québec », 38-39, 106-108.

78 Joseph Cauchon, «D'un traité élémentaire de physique, à l'usage des écoles et des familles ", Le Canadien, 2 décembre 1840.

79 Gagnon, "Les discours sur l'enseignement pratique ", 19-36.

80 Jean-Baptiste Meilleur, premier surintendant de l'éducation, est au préalable l'auteur d'un traité de chimie, Cours abrégé de leçons de chymie. Voir Léon Lortie, "Notes sur le 'cours abrégé de leçons de chymie' de Jean-Baptiste Meilleur ", Annales de l'Acfas 3 (1937) : 237-265.

81 Voir le site de Paul Aubin sur les manuels scolaires, www.bibl.ulaval.ca/ress/manscol, rubrique : dictionnaire des auteurs. 